\title{
Congenital oesophageal hiatal hernia in a pug
}

Keeley, B. ${ }^{1}$, Puggioni, A. ${ }^{1}$ and Pratschke, K. ${ }^{2}$ 1 School of Agriculture, Food Science and Veterinary Medicine, College of Life Sciences, University College
Dublin, Belfield, Dublin 4

2 Small Animal Hospital, University of Glasgow, Garscube Estate, 463 Bearsden Road, Glasgow G61 1QH

\begin{abstract}
Congenital sliding, oesophageal or type I hiatal hernia was diagnosed in a five-month-old pug puppy presented for evaluation of dyspnoea post feeding. The diagnosis was confirmed using plain film radiography. Surgical reduction of the hernia followed by plication of the oesophageal hiatus, oesophagopexy and left flank gastropexy permitted restoration of normal function. At 12 months of age, the dog was asymptomatic. This article describes the diagnosis and treatment of a congenital type 1 oesophageal hiatal herniation with unusual clinical presentation.
\end{abstract}

Key Words: congenital, dog, hiatal hernia, oesophagus

Corresponding author:

Ben Keeley

31 Anna Villas

Ranelagh

Dublin 6

Email: bjk78@hotmail.com

\section{Introduction}

A hiatal hernia is defined as any protrusion of abdominal contents through the oesophageal hiatus of the diaphragm into the thoracic cavity in the presence of an intact phrenico-oesophageal ligament (Pairolero, 1989). Hiatal herniation is thought to develop where there is an abnormality or laxity in the phrenico-oesophageal ligament (Prymak et al., 1989) that allows excessive movement of the oesophagus, gastro-oesophageal junction, gastric cardia and other abdominal organs.

Hiatal herniation was first recognised in the veterinary literature in 1974 (Gaskell et al.) and currently four types have been described in the dog and cat. Type I (sliding, axial or oesophageal) hiatal hernias are characterised by axial displacement of the distal part of the oesophagus, the gastro-oesophageal junction and part of the stomach through the oesophageal hiatus into the thoracic cavity, and is the most commonly reported type in small animals (Gaskell et al., 1974; Ellison et al., 1987; Bright et al., 1990; Callan et al., 1993; Pratschke et al., 1998). In type II (rolling or paraoesophageal) hernias, the distal segment of the oesophagus and lower oesophageal sphincter remain in a fixed position but a portion of the fundic region of the stomach herniates into the mediastinum alongside the thoracic oesophagus. Type II hiatal hernia is far less common and has been reported in three dogs but not in cats (Teunissen et al.,1978; Miles et al., 1988; Kirby et al., 2005). In type III, or mixed, hiatal hernia, characteristics of both types I and II are observed (Williams, 1990). Type IV hernia is a type III hernia complicated by the stomach or other abdominal viscera being located in the paraoesophageal sac (Auger and Riley, 1997; Rahal et al., 2003; Baig et al., 2006). Mixed (types III and IV) hiatal hernias are rare in small animals. It is important to differentiate between the types of hiatal hernia because the underlying pathology and pathophysiology are different, necessitating different treatments (Williams, 1990).

Clinical signs can be constant or, more commonly, intermittent due to the herniated organs moving back and forth from the abdominal to thoracic cavities. Reported clinical signs include regurgitation, coughing, anorexia, dyspnoea and hypersalivation (Lorinson and Bright, 1998). In the majority of cases reported to-date, clinical signs referable to secondary gastrointestinal dysfunction and/ or secondary gastro-oesophageal reflux disease (GORD) have predominated, in contrast to the case reported here where there were no recorded signs of GORD. This article describes the diagnosis and treatment of a congenital type 1 oesophageal hiatal herniation with unusual clinical presentation in a pug. To the authors' knowledge this is the first report of the condition in the pug.

\section{Case history}

A five-month-old female pug weighing $1.9 \mathrm{~kg}$ was referred to the University Veterinary Hospital, University College Dublin for evaluation of acute onset dyspnoea experienced immediately post-feeding. The dyspnoeic episodes were first noted five weeks prior to referral. The puppy had been fed a complete puppy kibble since weaning although elevated feeding of a soft gruel was initiated subsequent to the onset of clinical signs. Although this reduced the severity of clinical signs, it did not alleviate the problem. No vomiting, regurgitation or salivation had been noted. 


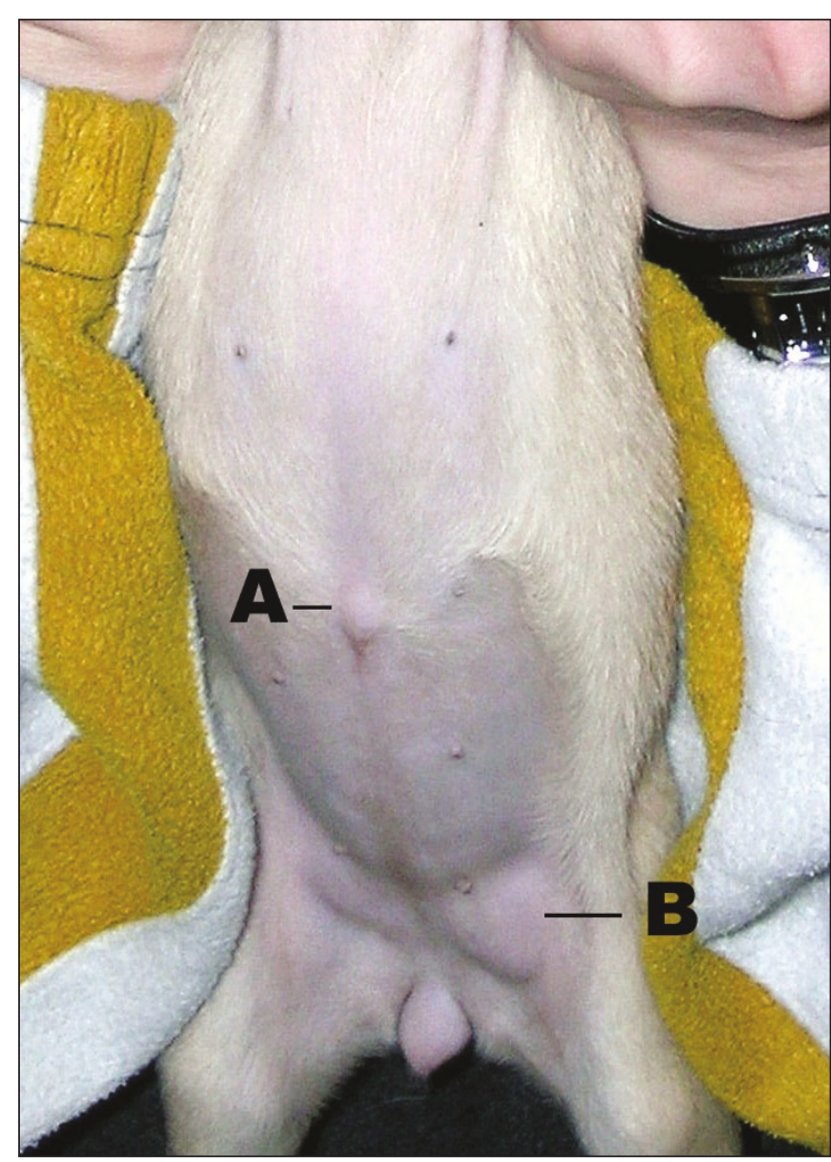

Figure 1: Photograph demonstrating the presence of an umbilical (A) and a left inguinal (B) hernia.

On initial examination, the puppy was bright, alert and responsive although body condition was only moderate. A $10 \mathrm{~mm}$ diameter umbilical hernia and a freely moveable $8 \mathrm{~mm}$ by $12 \mathrm{~mm}$ left inguinal hernia were found on abdominal palpation (Figure 1). Results of a complete blood cell count and biochemistry panel were within normal limits for the laboratory used.

The puppy was sedated with $3 \mathrm{mg} / \mathrm{kg}$ pethidine (Pethidine, Antigen Pharmaceuticals) and $0.25 \mathrm{mg} / \mathrm{kg}$ midazolam (Hypnoval, Roche) intramuscularly for radiographic investigation. Full body, lateromedial and ventrodorsal radiographs (Figure 2 and 3) demonstrated an oval soft tissue opacity in the midline of the caudodorsal thorax superimposed on the diaphragm, causing border effacement with the right crus. The opacity extended

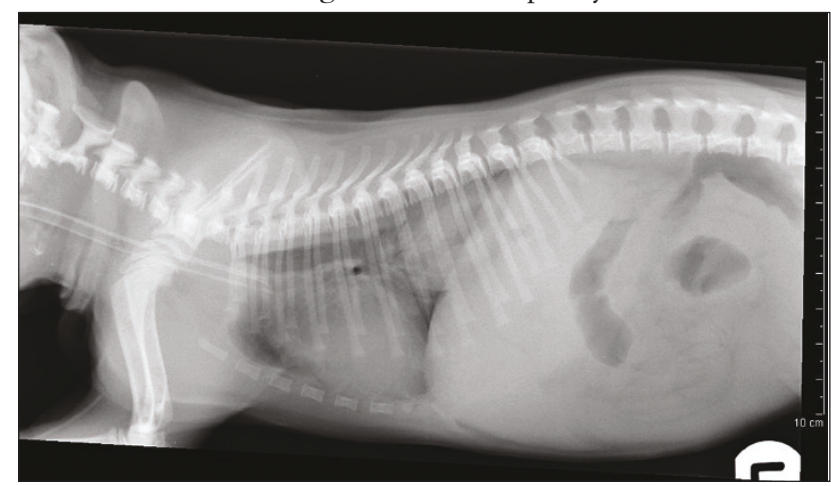

Figure 2: Left lateral thoracic radiograph demonstrating an oval soft tissue opacity in the caudodorsal thorax. The opacity extends cranially to the level of the heart base. The thoracic oesophagus is mildly distended and gas filled. cranially to the level of the heart base. A faint radiolucency of irregular shape was seen in the centre of the soft tissue mass, suggesting that the opacity was a partially herniated gastric fundus containing rugal folds. The intra-abdominal gas shadow of the gastric fundus could not be identified in either of the views. The thoracic oesophagus was mildly distended and gas filled. A positive contrast gastrointestinal study was not performed. The lung pattern was within normal limits with no evidence of aspiration pneumonia. Due to the persistence of clinical signs despite altering the feeding regime, and the fact that the predominant clinical sign was dyspnoea due to the space occupying effect of the hernia, surgical intervention was recommended. The puppy was premedicated with $3 \mathrm{mg} / \mathrm{kg}$ pethidine (Pethidine, Antigen Pharmaceuticals) and $0.25 \mathrm{mg} / \mathrm{kg}$ midazolam (Hypnoval, Roche) intramuscularly. General anaesthesia was induced with $4 \mathrm{mg} / \mathrm{kg}$ propofol (Rapinovet, Schering-Plough Animal Health Ltd.) intravenously and maintained with isofluorane (Forane, Abbott Laboratories Ltd) delivered in oxygen via endotracheal intubation. The soft palate, pharyngeal soft tissues and laryngeal function and appearance were visually assessed prior to endotracheal intubation to identify any concurrent brachycephalic obstructive airway syndrome. All parameters were within normal limits. The hernia was approached via a ventral midline coeliotomy incision extending from the xiphoid process to the pubis. A type I (sliding) hiatal hernia was identified with a subjectively lax phrenico-oesophageal ligament allowing the gastro-oesophageal junction and gastric cardia to lie within the thoracic cavity. The hernia was readily reducible with caudal traction; however, it recurred as soon as traction was released. The oesophageal hiatus measured approximately 2 $\mathrm{cm}$ in diameter but was otherwise normal in gross appearance.

Surgical reduction of the hernia followed by plication of the oesophageal hiatus, oesophagopexy and left flank gastropexy were performed. The liver was retracted to the right side of the abdomen while caudal traction was placed on the stomach and the hernia reduced satisfactorily. Two simple interrupted sutures were placed, using 2 metric polydioxanone (PDS; Ethicon), between the right and left crural muscles adjacent to the oesophageal hiatus and the corresponding ventrolateral aspect of the oesophagus. Care was taken not to include the dorsal or ventral vagal trunks in the sutures. The sutures engaged the seromuscular layers of the oesophagus and full thickness of the diaphragmatic crural muscle. The oesophageal hiatus was then plicated using three simple interrupted sutures of 2 metric polydioxanone (PDS, Ethicon) to reduce the diameter to within subjectively normal limits.

A $4 \mathrm{~cm}$ seromuscular incision was made in a cranial to caudal direction on the fundus of the stomach running parallel to the left craniolateral abdominal wall. A similar length incision was made on the lateral aspect of the left abdominal wall through the peritoneum and transversus abdominis muscle starting $1 \mathrm{~cm}$ caudal to, and running perpendicular with, the last rib to allow left flank incisional gastropexy. The free edges of the seromuscular 
incision and the abdominal wall incision were apposed with a single layer of simple continuous sutures using 2 metric polydioxanone (PDS; Ethicon). Completion of the gastropexy procedure resulted in sufficient caudal movement of the stomach to help maintain reduction of the hiatal hernia and also create a mild degree of traction at the gastro-oesophageal junction. Umbilical herniorraphy was performed as part of the coeliotomy closure, while a separate surgical approach was made to the left inguinal region and herniorrhaphy performed using horizontal mattress sutures of 2 metric polydioxanone (PDS; Ethicon). Although the puppy was not neutered at the time of surgery in accordance with the owner's wishes, they were strongly advised that she should not be used for breeding. The puppy recovered uneventfully from surgery, with postoperative analgesia provided by a combination of $0.2 \mathrm{mg} / \mathrm{kg}$ morphine sulphate intramuscularly every four hours for 24 hours (Morphine sulphate injection, Antigen Pharmaceuticals) and $0.1 \mathrm{mg} / \mathrm{kg}$ meloxicam by mouth every 24 hours for three days (Metacam, Boehringer Ingelheim). For the first two weeks post-operatively, the puppy was regularly fed small meals of a commercially available complete tinned puppy food from an elevated position. On re-examination two months post-surgery, no further episodes of dyspnoea had occurred and the puppy was eating and growing well.

A repeat upper airway examination was advised six months post-operatively although the owner declined. Followup telephone conversations with the owner at six and 12 months post-operatively indicated that the dog was in good general health, with a normal appetite and had suffered no further bouts of dyspnoea or other signs referable to either hiatal hernia or gastro-oesophageal reflux disease.

\section{Discussion}

Hiatal hernia was first described in the veterinary literature in 1974 by Gaskell and co-workers. Despite numerous additional cases having been reported since then, the true incidence, aetiology and pathophysiology of the disease remain incompletely identified. A number of interrelated factors, including: displacement of the lower oesophageal sphincter (LOS); changes in the angle of insertion of the oesophagus into the stomach; anatomical changes to the hiatal canal and phrenico-oesophageal ligament; oesophageal mobility disorders; underlying respiratory, neurological or neuromuscular disease; and others have been indicated in the aetiopathogenesis of this complex disease (Sivacolundhu et al., 2002).

A distinction has been made between congenital and acquired sliding hiatal hernias in the dog on the basis of age (Ellison et al., 1987). In congenital hiatal hernia, clinical signs may be observed immediately after weaning onto solid food and are usually seen before one year. Approximately $60 \%$ of cases of hiatal hernia reported in the veterinary literature are congenital (Guilford and Strombeck, 1996) occurring secondary to incomplete fusion of the diaphragm during early embryonic development (Callan et al., 1993). A familial inheritance (autosomal dominant) for sliding hiatal hernias has been shown in

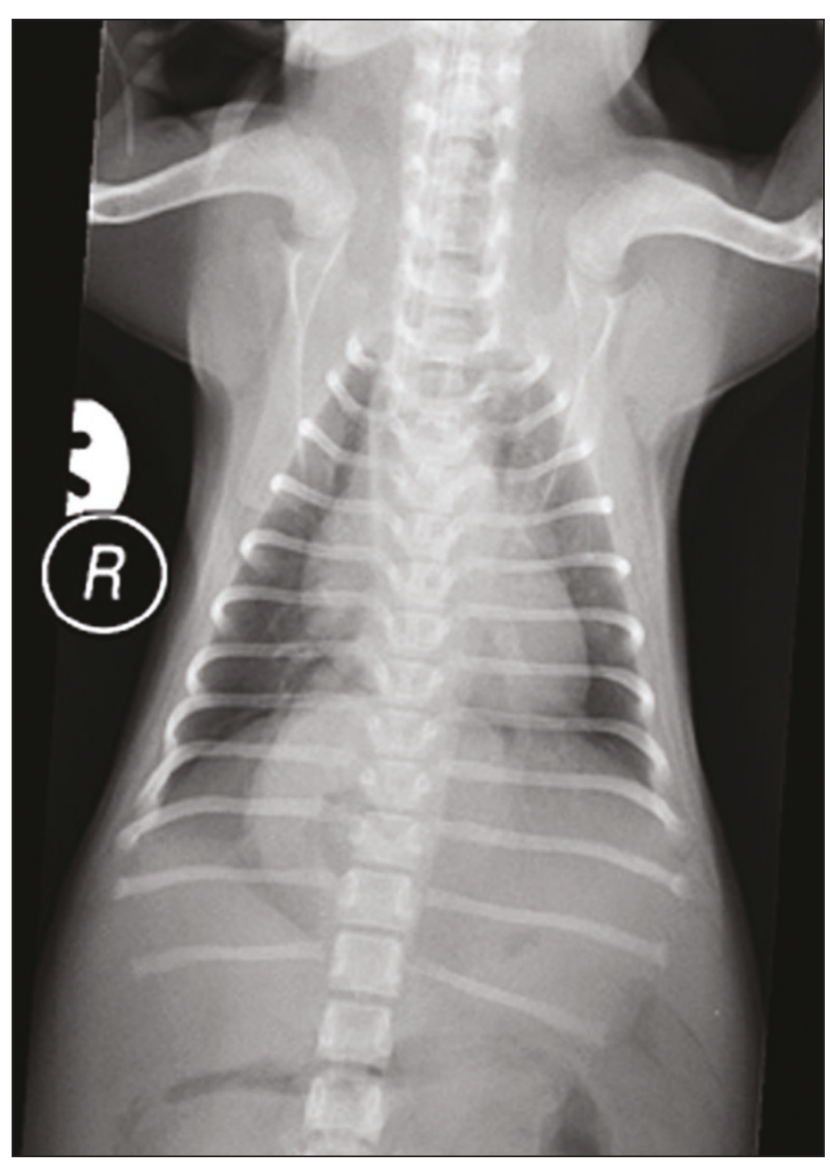

Figure 3: Dorsoventral thoracic radiograph demonstrating an oval soft tissue opacity in the caudal midline of the thorax.

humans (Carre et al., 1999). While the inheritance pattern has not been established for either type I or II hiatal hernias in dogs, it has been suggested that Shar-pei dogs may be predisposed to developing type I hernias (Callan et al., 1993). Interestingly, most of the reported cases of congenital hiatal hernia to-date have been in breeds classified as brachycephalic. Increased inspiratory effort secondary to brachycephalic airway syndrome, as with any upper airway obstructive disease, can result in an increase in negative intra-oesophageal and intrapleural pressure. This may then tend to pull the oesophagus and stomach into the thorax and exacerbate clinical signs related to hiatal herniation (Ellison et al., 1987; Burnie et al., 1989; Pratschke et al., 1998). Poncet et al. (2005; 2006) documented a strong correlation between brachycephalic airway syndrome and GORD. The authors proposed that gastrooesophageal reflex disorders aggravate respiratory signs by encumbering the pharyngeal region and stimulating persistent inflammation. Conversely, the chronic respiratory depression promotes gastro-oesophageal reflux.

GORD is one of the major consequences of hiatal hernia; indeed much of the symptomatology of clinically important hiatal hernia is thought to depend on concurrent GORD and reflux oesophagitis (Lorinson and Bright, 1998). Hypersalivation and vomiting or regurgitation, especially in the immediate postprandial period, is frequently reported (Prymak et al., 1989; Bright et al., 1990; Callan et al., 1993). Regurgitation and hypersalivation result from the chemical effects of gastric fluid (e.g., hydrogen ions and pepsins) on 
oesophageal mucosa, whereas vomiting can result from the obstructive effects of the hernia (Callan et al., 1993). Often, poor body condition results from this altered digestive function.

Dyspnoea and exercise intolerance have also been observed secondary to either aspiration pneumonia or lung compression by the herniated abdominal organs (Senyk et al., 1974; Pratschke et al., 1998). The presence of gastric acid in the lower oesophagus can result in severe laryngospasm and bronchospasm, which will worsen respiratory signs (Hardie et al., 1998). However, dyspnoea as a solitary presenting sign is unusual. Type I hiatal hernia can also be an incidental finding and it may be that many animals have asymptomatic hiatal herniation as seen in humans.

Survey radiography was diagnostic of hiatal hernia in this case; with the presence of a gas-filled intra-thoracic soft tissue structure containing gastric rugal folds within the caudo-dorsal thorax adjacent to the diaphragm. Additional thoracic radiographic abnormalities seen with hiatal hernia may include megaoesophagus, a solid soft tissue mass in the caudo-dorsal thorax adjacent to the diaphragm, absence of the right crus of the diaphragmatic border and lobar alveolar consolidation due to aspiration pneumonia (Ellison et al., 1987; Prymak et al., 1989). Although survey thoracic radiographs alone can sometimes diagnose hiatal hernia, it is frequently necessary to perform a positive contrast oesophagram or gastrogram to document the disorder (Kirby et al., 2005). This is particularly the case in patients with sliding hiatal hernia, in which the oesophagus and stomach may only intermittently be displaced. Fluoroscopy after oral administration of barium paste is one of the most reliable means of detecting intermittent hiatal hernia and assessing oesophageal motility (Hunt and Johnson, 2002). The use of abdominal ultrasonography has also been reported as an additional aid in the diagnosis of hiatal herniation (Dvir et al., 2003) and does not carry the risk of aspiration associated with the use of contrast material in a potentially dysphagic or regurgitating patient.

Symptomatic congenital hiatal hernias frequently require surgical correction although this should only be performed if conservative therapy is unsuccessful (Lorinson and Bright, 1998). The recommended regime for medical management is effectively the same as that described for gastro-oesophageal reflux, reflux oesophagitis and gastritis. This involves elevated feeding, reduction of gastric acid secretion with histamine- 2 antagonists such as cimetidine and ranitidine, protection of the oesophageal mucosa with sucralfate, and the use of prokinetic agents such as metoclopramide or cisapride to increase lower oesophageal sphincter tone (Prymak et al., 1989). In the current case the only clinical sign was dyspnoea after feeding, presumably due to the space-occupying effect of the hernia, with none of the typical signs of GORD reported. Therefore, it was not surprising that a conservative approach did not allow full resolution of clinical signs.

Surgical treatment of hiatal hernia is a focus of controversy in both the human and veterinary literature (Ellison $e t$ al., 1987; Prymak et al., 1989; Sivacolundhu et al., 2002).
Surgical treatment is aimed at achieving reduction of the hernia and restoration of normal LOS function (Williams, 1990). Restoration and stabilisation of what has been described as 'normal anatomy' by reducing the hernia, decreasing the size of the oesophageal hiatus and performing an oesophagopexy and gastropexy is an effective means of treating hiatal hernia in dogs and cats (Prymak et al., 1989; White, 1993). This combination of surgical procedures was first reported by Robotham (1979) and is based on the assumption that all animals have a distinct intra-abdominal segment of oesophagus creating an essential flutter valve that maintains gastrooesophageal barrier function. However, the presence of an abdominal oesophagus is not necessarily a normal finding in every dog and it has been proposed that oesophagopexy, phrenicoplasty and left flank gastropexy may not be so much about restoring normal anatomy as creation of an anatomical situation which artificially elevates pressure at the gastro-oesophageal junction and hence reinforces the anti-reflux barrier (Pratschke et al., 2001). Recent evaluation of the pathophysiology of hiatal hernia suggests that anti-reflux procedures (such as Nissen fundoplication) are not indicated in veterinary patients as a primary incompetence of the lower oesophageal sphincter rarely exists (Sivacolundhu et al., 2002). The use of anti-reflux procedures in small animals has been widely discontinued due to the unacceptably high risk of complications, including dysphagia, 'gas bloat' syndrome, the inability to belch and reherniation, which may lead to compromise of the stomach wall and death (Ellison et al., 1987). With attention to exacerbating problems such as brachycephalic syndrome, good results may be expected with treatment of hiatal hernia (Prymak et al., 1989; White, 1993).

\section{References}

Auger, J. M. and Riley, S. M. (1997). Combined hiatal and pleuroperitoneal hernia in a Shar-Pei. Canadian Veterinary Journal 38: 640-642.

Baig, M. A., Gemmill, T., Hammond, G., Patterson, C. and Ramsey, I.

(2006). Budd-Chiari-like syndrome caused by a congenital hiatal hernia in a Shar-Pei dog. The Veterinary Record, 159: 322-323.

Bright, R. M., Sackman, J. E., Denovo, C. and Toal, C. (1990). Hiatal

hernia in the dog and cat: a retrospective study of 16 cases. Journal of Small Animal Practice 31: 244-250.

Burnie, A. G., Simpson, J. W. and Corcoran, B. M. (1989). Gastro-

oesophageal reflux and hiatus hernia associated with laryngeal paralysis in a dog. Journal of Small Animal Practice 30: 414-416.

Callan, M. B., Washabau, R. J., Saunders, H. M., Kerr, L., Prymak, C. and Holt, D. (1993). Congenital esophageal hiatal hernia in the Chinese SharPei dog. Journal of Veterinary Internal Medicine 7: 210-215.

Carre, I. J., Johnston, B. T., Thomas, P. S. and Morrison, P. J. (1999).

Familial hiatal hernia in a large five-generation family confirming true autosomal dominant inheritance. Gut 45: 649-652.

Dvir, E., Spotswood, T. C., Lambrechts, N. E. and Lobetti, R. G. (2003). Congenital narrowing of the intrapharyngeal opeing in a dog with concurrent oesophageal hiatal hernia. Journal of Small Animal Practice 44: 359-362

Ellison, G. W., Lewis, D. D., Phillips, L and Tarvin, G. (1987). Esophageal hiatal hernia in small animals: literature review and a modified surgical technique. Journal of the American Animal Hospital Association 23: 391-399. Gaskell, C. J., Gibbs, C. and Pearson, H. (1974). Sliding hiatus hernia with reflux oesophagitis in two dogs. Journal of Small Animal Practice 15(8): 
503-509.

Guilford, W. G. and Strombeck, D. R. (1996). Diseases of swallowing. In: Strombeck's Small Animal Gastroenterology, $3^{\text {rd }}$ edn. Eds W. G.

Guilford, S. A. Center, D. R. Strombeck, D. A. Williams and D. J. Meyer. Philadelphia. pp 211-238.

Hardie, E. M., Ramirez, O., Clary, E. M., Kornegay, J. N., Correa, M. T., Feimster, R. A. and Robertson, E. R. (1998). Abnormalities of the thoracic bellows: stress fractures of the ribs and hiatal hernia. Journal of Veterinary Internal Medicine 12: 279-287.

Hunt, G. B. and Johnson, K. A. (2002). Diaphragmatic, pericardial, and hiatal hernia. In: Textbook of Small Animal Surgery, $3^{\text {rd }}$ edn. Ed D. H. Slatter. W. B. Saunders, Philadelphia pp 471-487.

Kirby, K. A., Bright, R. M. and Owen, H. D. (2005). Paraoesophageal hiatal hernia and megaoesophagus in a three-week-old Alaskan malamute. Journal of Small Animal Practice 46: 402-405.

Lorinson, D. and Bright, R. M. (1998); Long-term outcome of medical and surgical treatment of hiatal hernias in dogs and cats: 27 cases (1978-1996). Journal of the American Veterinary Medical Association 213: 381-383;

Miles, K. G., Pope, E. R. and Jergens, A. E. (1988). Paraesophageal hiatal hernia and pyloric obstruction in a dog. Journal of the American Veterinary Medical Association 193: 1437-1439.

Pairolero, P. C. (1989). Oesophagus and diaphragmatic hernias. In Schwartz SI, et al (eds): Principles of Surgery, $5^{\text {th }}$ ed. McGraw-Hill, New York.

Poncet, C. M., Dupre, G. P., Freiche, V. G., Estrada, M. M., Poubanne, Y. A. and Bouvy, B. M. (2005). Prevalence of gastrointestinal tract lesions in 73 brachycephalic dogs with upper respiratory syndrome. Journal of Small Animal Practice 46: 273-279.

Poncet, C. M., Dupre, G. P., Freiche, V. G. and Bouvy, B. M. (2006). Longterm results of upper respiratory syndrome surgery and gastro-intestinal tract medical treatment in 51 brachycephalic dogs. Journal of Small Animal Practice 47: 137-142.

Pratschke, K. M., Hughes, J. M. L., Skelly, C. and Bellenger, C. R. (1998). Hiatal herniation as a complication of chronic diaphragmatic herniation. Journal of Small Animal Practice 39: 33-38.

Pratschke, K. M., Bellenger, C. R., McAllister, H. and Campion, D. (2001). Barrier pressure at the gastroesophageal junction in anaesthetized dogs. American Journal of Veterinary Radiology vol 62 no. 7 pp 1068-1072.

Prymak, C., Saunders, H. M. and Washabau, R. J. (1989). Hiatal hernia repair by restoration and stabilization of normal anatomy: an evaluation of four dogs and one cat. Veterinary Surgery 18: 386-391.

Rahal, S. C., Mamprim, M. J., Muniz, L. M. R. and Teixeira, C. R. (2003). Type-4 Oesophageal hiatal hernia in a Chinese Shar-Pei dog. Veterinary Radiology and Ultrasound, Vol. 44, No. 6, pp 646-647.

Robotham. G. R. (1979). Congenital hiatal hernia in a cat. Feline Practice 9, 37-39

Senyk, J., Arorelius, M., Lilja, B. and Nylander, G. (1974). Bronchographic and angiopneumographic changes associated with oesophageal hiatus hernia. An experimental study in dogs. Eur Surg Res 6: 72-78. Sivacolundhu, R. K., Read, R. A. and Marchevsky, A. M. (2002). Hiatal hernia controversies - a review of pathophysiology and treatment options. Australian Veterinary Journal 80: 48-53.

Teunissen. G. H. B., Harpe, R. P., Van Toorenburg, J. and Wolvekamp, W. T. C. (1978). Esophageal hiatal hernia. Case report of a dog and a cheetah. Tijdschrift voor Diergeneeskunde 103: 742-749.

White, R. N. (1993). A modified technique for surgical repair of oesophageal hiatal herniation in the dog. Journal of Small Animal Practice 34: 599-603.

Williams, J. M. (1990). Hiatal hernia in a Shar-Pei. Journal of Small Animal Practice 31: 251-254. 